In all cases a trace of tannin could be detected on further extracting the materials.

The results given in this paper confirm the conclusions previously drawn that the continuous extractor has given the most complete extraction and that by this apparatus all soluble constituents are removed in larger quantities than by other extractors. It may be said, however, that while we believe this to be the best extractor with which we are acquainted, it is not to be doubted that other forms can be made to give as complete extraction, but at a greater expenditure of money, time, and labor.

We desire especially to call attention to the necessity for conplete extraction. With the comparatively large quantity of material that is used it is exceedingly difficult to secure this. All materials should be ground to pass at most a millimeter sieve, and extraction should be continued for at least sixteen hours, preferably for twenty-four hours. The efforts of this laboratory are now being directed toward so modifying the methods of tannin analysis that smaller quantities of material may be used and thus secure more complete extraction.

[CONTRIBUTION FROM THE BUREAU OF CHEMISTRY, U. S. DEPARTMENT OF Agriculture. PUblished by Permission of the SecreTARY OF AGRICULTURE. SENT BY H. W. WILEY.]

\title{
THE EXAMINATION OF WRITING INKS.1
}

\author{
BY L. S. MuNson.
}

Received January 18, เ906.

In many branches of the government service as well as in many state and private institutions it is frequently of particular importance that permanent records be preserved, and from necessity or convenience many of these are written records. It is well known that many of the old writing fluids yielded records that have remained unchanged for generations, and undoubtedly many of the writing inks in use at the present time are equally resistant. However, from the great advances made in recent years in the manufacture of colors has arisen the tendency to substitute these colors in whole or in part in the manufacture of ink. This investigation was taken up, therefore, to determine to what extent the inks in use in the different executive departments were suitable for record purposes.

1 Read at the New Orleans Meeting of the American Chemical Society. 
A total of thirty samples was examined. These represented eighteen distinct brands of ink and were the products of eight manufacturers. Three of these samples were for use expressly as copying inks, three were combined writing and copying inks, and of the remaining twenty-four the claim was made with eight that they would furnish good copies, especially when fresh. Twenty-seven of the samples were iron-tannin inks and three were chromate-logwood inks. There are but few of the latter class of inks upon the market, so this work had to do principally with the iron-tannin inks. These latter depend upon the tannate of iron which they contain for their permanence, and the principal ingredients entering into their composition are tannic acid, or a mixture of tannic and gallic acids, ferrous sulphate, a small amount of acid to keep the tannate of iron in solution, sufficient soluble dye to give the immediate color to the ink, and a small amount of preservative to prevent decomposition or the development of moulds. With such an ink the full development of the color takes place only after exposure of the writing to the air for a period of several days, hence the necessity of a considerable amount of soluble dye to give the immediate color. In case the ink is intended for copying purposes some hygroscopic material is added to facilitate this purpose.

The work on these inks included (I) chemical analysis of the ink; (2) the effect of exposure to sunlight and of treatment with reagents upon stripes made with the ink; and (3) the nature of the copy furnished, the effect upon this copy of exposure to sunlight, and the effect upon the original writing of taking press copies from the same. Full results of analysis and of the various tests applied are given in the accompanying table. Samples Nos. I 85 I, 1965 and 1986 were copying inks, Nos. I 720, I 964 and 1985 were combined writing and copying inks, and of the remaining samples the claim was made for Nos. 1962, I330, I7x7, I961, I $722,1329,1727$ and 1732 that they would yield good copies from the fresh writing.

Discussion of Results.-The specific gravity, total solids, ash, iron and sulphates were determined on all the samples except the logwood inks, in which cases instead of iron, potassium dichromate was determined. Of these, specific gravity and iron are considered of particular importance in determining the quality of 
an ink. The specific gravity of the copying inks ranged from I.0634 to I. I093, of the combined writing and copying inks from 1.0473 to 1.0493 , and of the writing inks from 1.0068 to $\mathrm{I} .04 \mathrm{II}$. The percentage of iron ranged in the copying inks from 1.46 to I. 80 , in the combined writing and copying inks from 0.73 to 0.80 , and in the writing inks from 0.05 to 0.68 . Of the copying inks, but two were iron-tannin inks, so the range given above for iron is not to be considered representative of this class of inks. In fact, the two iron-tannin inks examined were particularly heavy inks. One copying ink was a chromate-logwood ink containing 0.06 per cent. of potassium dichromate, and two writing inks were chromate-logwood inks containing 0.73 and 0.74 per cent. of potassium dichromate.

It is seen from the above that there are distinct differences in composition between the various classes of ink, and wide ranges between inks of the same class. With many writing inks permanence is not an essential feature, and in such cases the lighter and cheaper inks may serve the purpose as well as the heavier and more expensive ones. The results obtained in this investigation enable one to distinguish those inks that may be relied upon to produce permanent records, from those the writings of which are not permanent. By a permanent ink is meant one the writing of which, when exposed to the usual conditions of moderate heat, atmospheric moisture and sunlight, will resist destruction. It is well known that the iron-tannin inks as well as the chromatelogwood inks are easily destroyed by various chemical agents, notably acids, oxidizing agents, etc.

Upon exposure to sunlight and treatment with reagents the various inks showed marked differences in behavior. For these tests stripes were made upon good quality of paper by allowing the ink to flow from a pipette inclined at an angle of $45^{\circ}$ with the paper, using (I) the undiluted ink and (2) the ink diluted with an equal volume of water. Upon exposure of the stripes to sunlight, valuable information was obtained regarding the lasting qualities of the inks. When the stripes made with the undiluted ink were exposed for a period of twenty days a number of the lighter inks showed marked deterioration, and when the time of exposure was increased to eighty days all the iron-tannin inks containing less than 0.30 per cent. of iron, and all the chromate-logwood inks were badly faded, while a number of the iron-tannin inks 
containing more than 0.30 per cent. of iron showed marked deterioration. Stripes made with the diluted ink were much more readily affected, but a number remained unaffected after eighty days' exposure. Samples Nos. I7I8, I850, I720, I964, $1985,185 \mathrm{I}, \mathrm{I} 986, \mathrm{I} 96 \mathrm{I}$ and $143 \mathrm{I}$ showed little or no change when submitted to this test and are the only inks that are considered entirely satisfactory for record purposes.

When treated with calcium hypochlorite of a strength corresponding to $\mathrm{N} / 200$ chlorine the color of the ink was entirely destroyed in all cases, the length of time required depending largely upon the quality of the ink. In a number of cases, notably with the lighter inks, the color was entirely destroyed in less than fifteen minutes, while with the heaviest inks the time required was from one to two hours. This test, of course, gives only comparative results, but is of value as a ready means of determining the relative values of a number of inks.

Upon treating the stripes with water and with 95 per cent. alcohol a number of the inks showed marked loss of color, notably those containing small amounts of tannate of iron, and depending largely for their coloring power upon the soluble dye present in them. A good ink should undergo practically no change upon treating with water or with alcohol.

For the purpose of studying the nature and permanence of the copy furnished by the different inks, one copy was taken from the fresh writing and one copy from writing two days old. In nearly all cases the copy obtained from the writing two days old was as good as that taken from the fresh writing, but to obtain such a copy it was necessary to use somewhat moister pads and to leave the copy in press longer than with fresh writing. Only the heavy copying inks gave copies that were at all permanent when exposed to sunlight. With many the copy faded almost completely upon twenty days' exposure. This rapid fading of the copy is due in part to the small amount of ink that is actually transferred to and retained by the copy. While the writing may, and generally does, give up a considerable quantity of ink when the copy is taken, much of this passes into the moist pad and but little is actually retained by the light, porous paper upon which the copy is generally taken. Besides, an ink may readily give up the soluble dye, while but little tannate of iron is trans- 
ferred to the copy. In such case the power of resistance of the copy is very low.

The writing after press copying was exposed to sunlight along with the writing from which no press copy had been taken. In nearly all cases the former faded much more readily, and in some cases the difference was exceedingly marked, the greatest difference being found in the lighter inks.

The inks examined represent the products of nearly all the large manufacturers in this country and show a very wide range in composition. Many of these are unsuited for record purposes while several are entirely satisfactory as record inks.

The United States Treasury Department requires that the ink furnished that department shall equal in quality an ink made up with 23.4 grams tannic acid, 7.7 grams gallic acid, 30 grams ferrous sulphate, 10 grams gum arabic, $25 \mathrm{cc}$. dilute hydrochloric acid and I gram carbolic acid, the whole being made up in volume to rooo cc. A number of the inks examined were apparently made up to meet the above requirement. Such an ink would have a specific gravity of about 1.0360 and contain about 0.60 per cent. of iron, and would meet all ordinary requirement of a record ink. In fact, a few inks that were somewhat lighter than the above were found entirely satisfactory for record purposes. It is well, however, to have the requirements of such an ink amply high so long as its working qualities are not interfered with.

CONTRACTS IABORATORY.

\section{NITROSYL SELENIC ACID.}

By Victor LENHER AND J. H. Mathews.

Received Jatuary 27 , I906.

NrTROGEN tetroxide, when brought in contact with sulphuric acid, yields the well-known nitrosyl sulphuric acid. When selenic acid is substituted for the sulphuric acid, a product is obtained analogous to nitrosyl sulphuric acid. However, in the case of the preparation of nitrosyl selenic acid, it is necessary that a low temperature should be used.

When selenic acid having a strength of 83 per cent. is mixed with an excess of liquid nitrogen tetroxide and the solution cooled with solid carbon dioxide, a light blue solid forms on the surface, and immediately below this a layer of a darker blue solid. These two layers form over a heavy syrupy liquid, consisting 